\title{
Association Between Triglyceride Glucose Index and Carotid Artery Plaque in Different Glucose Metabolic States in Patients with Coronary Heart Disease - A RCSCD-TCM Study in China
}

\section{Zhu Li}

Tianjin University of Traditional Chinese Medicine

\section{Yuanyuan He}

Tianjin University of Traditional Chinese Medicine

\section{Shuo Wang}

Tianjin University of Traditional Chinese Medicine

\section{Lin Li}

Tianjin University of Traditional Chinese Medicine

\section{Rongrong Yang}

Tianjin University of Traditional Chinese Medicine

Yijia Liu

Tianjin University of Traditional Chinese Medicine

\section{Qi Cheng}

Tianjin University of Traditional Chinese Medicine

Lu Yu

Tianjin University of Traditional Chinese Medicine

Yanchao Zheng

Tianjin University of Traditional Chinese Medicine

Hongmei Zheng

Tianjin Medical University General Hospital

\section{Shan Gao}

Tianjin University of Traditional Chinese Medicine

Chunquan Yu ( $\nabla$ ycqtjutcm@foxmail.com )

Tianjin University of Traditional Chinese Medicine

\section{Research Article}

Keywords: Triglyceride, Fasting plasma glucose, TyG index, Coronary heart disease, Carotid plaques

Posted Date: January 12th, 2022

DOl: https://doi.org/10.21203/rs.3.rs-1235048/v1 
License: (a) (i) This work is licensed under a Creative Commons Attribution 4.0 International License. Read Full License

Version of Record: A version of this preprint was published at Cardiovascular Diabetology on March 11th, 2022. See the published version at https://doi.org/10.1186/s12933-022-01470-3. 


\section{Abstract}

\section{Background}

The triglyceride glucose $(T y G)$ index serves as a surrogate indicator of insulin resistance. However, there are limited data on the association between TyG index and carotid artery plaque (CAP) in patients with coronary heart disease (CHD).

\section{Methods}

A total of 10,535 CHD patients were included in this study. TyG index was divided into quartiles, Q1: TyG index $\nabla$ 8.52, Q2: $8.52 \leq$ TyG index $\otimes 8.93$, Q3: $8.93 \leq$ TyG index $\leq 9.40$, Q4: TyG index $\otimes 9.40$. Logistic regression was used to analyze the relationship between TyG index and CAP in CHD patients, and further analyzed the relationship between TyG index and CAP in different genders, different age groups and different glucose metabolism states.

\section{Results}

A baseline analysis of CHD patients divided into four groups according to the quartile of the TyG index showed that there were significant differences in related parameters between the groups. As the TyG index increases, the incidence of CAP increases significantly. After adjustment for multivariate, TyG index levels for Q3 and Q4 correlated with increased OR in CAP, which Q4 has the highest correlation (OR: 1.42; 95\% Cl: $1.33-1.53$ ). The correlation between the Tyg index of female (OR:1.38; $95 \% \mathrm{Cl}: 1.31-1.45)$ and CAP was higher than that of male (OR:1.23; 95\% Cl: 1.16, 1.30). The OR value of middle-aged ( $\leq 60$ years old) patients (OR:1.14; 95\% Cl: 1.071.22 ) is higher than that of elderly (खdayu 60 years old) patients (OR:1.07; $95 \% \mathrm{Cl}: 1.02-1.13$ ). Under different glucose metabolism states, the TyG index of CHD patients was significantly related to the risk of CAP, and the OR value of diabetes (DM) was the highest (OR: 1.35; 95\% Cl: 1.26-1.45).

\section{Conclusions}

There is a significant correlation between the TyG index and CAP in CHD patients. In addition, the correlation between TyG index and CAP in CHD patients is higher in female than in male, and the correlation in middle-aged and elderly patients is higher than that in elderly patients. Under the condition of $\mathrm{DM}$, the correlation between TyG index and carotid artery plaque in CHD patients is higher.

\section{Background}

Coronary heart disease (CHD) is class of chronic non-communicable diseases (NCD) with extremely high incidence and mortality. Diabetes (DM) usually coexists with arterial hypertension and dyslipidemia [1-2]. Type 2 diabetes (T2DM) accounts for $95 \%$ of the diseases diagnosed as DM. Which is one of the risk factors for coronary artery disease (CAD) and the progression and rupture of atherosclerotic plaque [3]. CAD is also a common comorbidities and major cause of death in DM patients. Studies have shown that in participants without CHD history, T2DM is associated with carotid artery plaque (CAP), which is a better predictor than high carotid artery intima-media thickness (CIMT) indicators or recurrent cardiovascular events [4]. Pre-DM patients 
have a high propensity for to develop DM [5]. Many studies have reported that CHD patients have a higher risk of adverse prognosis during their Pre-DM and glucose metabolism disorders [6-8].

The triglyceride glucose (TyG) index is a valuable biomarker for the development of diabetes, and the TyG index is used as a marker of insulin resistance (IR), leading to the occurrence of NCD [9-10]. Many studies have shown that the TyG index is related to the high prevalence of CAD and the increased risk of major adverse cardiovascular and cerebrovascular events [11-12], including ischemic stroke [13], increased arterial stiffness [14-16], hypertension [17], coronary artery stenosis [18], and carotid atherosclerosis (AS) [19] are related to the morbidity. However, there is no relevant research to investigate the correlation between TyG index and CAP in CHD patients under different glucose metabolism status.

Therefore, the purpose of this study was to clarify the correlation of TyG index and CAP in the different glucose metabolic states of CHD patients, and to further investigate the correlation of TyG index and CAP in the different stratification of gender and age. It is hoped that in the clinical treatment of $\mathrm{CHD}$, through the identification of simpler biochemical indicators, to prevent the risk of AS (such as CAP).

\section{Methods}

\section{Patients}

This study is a large-scale, multi-center retrospective cohort study. The participants included 107,301 CHD inpatients who were admitted to 6 hospitals in Tianjin from January 1, 2014 to September 30, 2020. The root investigation study design excluded age less than 35years or older than 80 years, patients with tumor, infectious, or severe liver or kidney disease, and patients lacking data on TG, FPG, and carotid ultrasound measurements. A total of 10,535 participants were eventually included in the study. A flowchart of the patients recruitment was shown in Figure 1. This study was approved by the ethics committee of the Tianjin University of Traditional Chinese Medicine (TJUTCM-EC20190008) and registered with the Chinese Clinical Trial Registry (ChiCTR-1900024535) and in ClinicalTrials.gov (NCT04026724).

\section{Data collection}

In this study, age, gender, smoking, drinking, and medication history of patients were recorded through standard structured questionnaires [20,21]. Systolic blood pressure (SBP) and diastolic blood pressure (DBP) were measured by experienced technicians at the heart level using automatic blood pressure monitors. SBP $\geq 130$ $\mathrm{mmHg}$ or a DBP $\geq 80 \mathrm{mmHg}$ was defined as hypertension [22].

All participants collected fasting venous blood samples in early morning. The fasting plasma glucose (FPG), total cholesterol (TC), high-density lipoprotein cholesterol (HDL-C), and triglycerides (TG), low-density lipoprotein cholesterol (LDL-C), C-reactionprotein (CRP), glycated haemoglobin (HbA1c) were measured on automatic haematology analyser. And strictly follow the laboratory standard procedures for quality control. TyG index was calculated as $\mathrm{Ln}[\mathrm{fasting}$ triglycerides $(\mathrm{mg} / \mathrm{dl}) \times$ fasting glucose $(\mathrm{mg} / \mathrm{dl}) / 2]$ [23]. Hyperlipidemia was defined as TC $\geq 6.2 \mathrm{mmol} / \mathrm{L}(240 \mathrm{mg} / \mathrm{dL}), \mathrm{TG} \geq 2.3 \mathrm{mmol} / \mathrm{L}(200 \mathrm{mg} / \mathrm{dL}), \mathrm{LDL}-\mathrm{C} \geq 4.1 \mathrm{mmol} / \mathrm{L}(160 \mathrm{mg} / \mathrm{dL})$, or $\mathrm{HDL}-\mathrm{C} \leq 1.0 \mathrm{mmol} / \mathrm{L}(40 \mathrm{mg} / \mathrm{dL})[24,25]$. 
Carotid ultrasound examination was performed by certified professional technician using a diagnostic ultrasound system. In B-mode imaging, scan and image the common carotid artery, internal carotid artery, and carotid artery bifurcation. The CIMT was defined as the average value of the IMT of the left and right common carotid arteries [26]. Professional doctors analyzed the color of the carotid artery based on the Doppler ultrasound results and recorded the number of CAP and echo characteristics. The number of CAP was divided into single $(n=1)$ and multiple $(n \geq 2)$. The echogenic properties of CAP were divided hypoechoic, isoechoic, hyperechoic, and mixed. This study employed rigorous quality control procedures to maintain consistency in monitoring and test image acquisition and analysis. Interlaboratory quality were all assessmented by licensed experimenters.

\section{Statistical analyses}

The characteristics of participants in the different groups were compared using $\chi^{2}$ tests, $t$-test and the MannWhitney test. The odds ratios (ORs) and 95\% confidence intervals (Cls) of CAP were estimated for TyG index using logistic regressions. Age, sex, SBP, DBP, CRP, HbA1c, TC, HDL-C, LDL-C, smoking, drinking, hypertension, hyperlipidemia, use of antihypertensives, use of antilipidemic were considered potential confounders in this association. The collinearity of different models was tested before logstic regressions. Missing values are imputed by multiple imputation method. All statistical analyses were performed using SPSS 24.0 (IBM Corp, New York, NY, USA).

\section{Results}

\section{Baseline characteristics}

The basic characteristics of the study population of 10,535 cases were shown in Table 1. The average age of the participants was 64 years old, and the proportion of female (51.4\%) was slightly more than that of male (48.6\%). Among them, 8079 (76.69\%) patients had CAP, and the highest proportion of DM patients was $37.6 \%$. All subjects were divided into 4 groups according to the quartile level of TyG index, variables were significantly different between the groups. Generally speaking, DBP, SBP, FPG, TC, TG, HbA1c, smoking, drinking, hyperlipidemia, use of antihypertensives, use of antilipidemic were positively correlated with the quartile level of TyG, while HDL-C was positively correlated with the quartile level of TyG index, however HDL-C negatively correlated with the quartile level of TyG index.

\section{Table 1. General characteristics of study participants according to TyG index}


Characteristic

TyG index

$P$-value

$\begin{array}{lllll}\text { Total } & \text { Q1 } & \text { Q2 } & \text { Q3 } & \text { Q4 }\end{array}$

$(N=10535)$

$\nabla n=2650 \rrbracket \quad \nabla n=2647 \rrbracket \quad \nabla n=2590 \rrbracket \quad \nabla n=2648 \rrbracket$

Sex, n (\%)

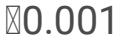

male

$5121(48.6)$

1375 (51.9)

$1256(47.4)$

$1183(45.7)$

1307 (49.4)

Female

$5414(51.4)$

$1275(48.1)$

$1391(52.6)$

1407 (54.3) $1341(50.6)$

Age, years, median

(IQR)

Total

$64.0(59.0-$

70.0)

$65.0(60.0-\quad 64.0$ (59.0-

69.0)

$64.0(59.0$

$63.0(57.0$

71.0

$55.0(52.0-$

$55.0(51.0$

$55.0(51.0-$

58.0)

58.0)

58.0)

69.0)

68.0)

$\leq 60$

$67.0(64.0$
$71.0)$

$68.0(64.0-$

$67.0(64.0$

$55.0(52.0-$

58.0)

$55.0(51.0-$

$\llbracket 60$

72.0)

71.0)

$67.0(63.0-$

71.0)

$57.0)$

SBP, $\mathrm{mmHg}$,

median (IQR)

140.0

(128.0-

139.0

156.0)

(124.0-

140.0

(126.0-

152.0)

155.0)

140.0

(130.0-

159.0)

$67.0(64.0-$

71.0)

DBP, $m m H g$,

83.0 (77.0-

80.0 (75.0-

$82.0(76.0$

83.0 (78.0-

90.0)

90.0)

90.0)

141.0

(130.0-

159.0)

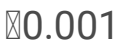

CRP, mg/L, median

4.2 (2.0-

4.1 (1.9-

4.3 (2.0-

$4.0(2.0-$

11.3)

84.0 (78.0- $\quad \varangle 0.001$

12.8)

14.9)

14.2)

FPG, $\mathrm{mmol} / \mathrm{L}$,

median (IQR)

$6.2(5.3-8.1)$

$5.3(4.8-5.9) \quad 5.8(5.1-6.8)$

6.54 (5.6-

8.1)

9.4 (7.0-

12.6)

4.5 (2.2-

$\bigotimes 0.001$

LDL-C, mmol/L,

$2.8(2.1-3.4)$

$2.5(1.9-3.1)$

$2.8(2.2-3.4)$

$3.0(2.3-3.6)$

$2.8(2.2-3.5) \quad \nabla 0.001$

median (IQR)

HDL-C, mmol/L,

$1.1(0.9-1.3)$

$1.2(1.0-1.4)$

$1.1(0.9-1.3)$

$1.1(0.9-1.2)$

$1.0(0.8-1.1) \quad \varangle 0.001$

median (IQR)

TG, mmol/L,

median (IQR)

$1.4(1.0-2.1)$

$0.9(0.7-1.0)$

$1.3(1.1-1.5)$

$1.8(1.5-2.1)$

$2.6(2.0-3.5)$

$\varangle 0.001$

TC, mmol/L,

median (IQR)

$4.6(3.8-5.4)$

$4.1(3.4-4.9) \quad 4.5(3.8-5.2)$

$4.7(4.0-5.5)$

$5.0(4.2-5.7)$

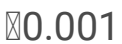

HbA1c, mmol/L

median (IQR)

$6.0(5.6-7.0)$

$5.7(5.4-6.2) \quad 5.9(5.5-6.5)$

$6.1(5.6-6.9)$

$7.1(6.1-8.6)$

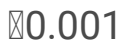

TyG index

$9.0(8.5-9.4)$

$8.2(8.0-8.4)$

$8.7(8.6-8.8)$

$9.1(9.0-9.3)$

$9.8(9.6-$

10.2)

$\mathbb{\nabla} 0.001$

\begin{tabular}{|c|c|c|c|c|c|c|}
\hline smoking, n (\%) & $4590(43.6)$ & 1173 (44.3) & $1121(42.3)$ & $1101(42.5)$ & $1195(45.1)$ & $\varangle 0.001$ \\
\hline Drinking, n (\%) & $5863(55.7)$ & $1457(55)$ & 1480 (55.9) & 1421 (54.9) & 1505 (56.8) & $\varangle 0.001$ \\
\hline Hypertension, n (\%) & $4297(40.8)$ & 1041 (39.3) & 1072 (40.5) & 1107 (42.7) & 1077 (40.7) & $\llbracket 0.001$ \\
\hline Hyperlipidemia, n & $1039(9.9)$ & $149(5.6)$ & $196(7.4)$ & $270(10.4)$ & $424(16)$ & $\varangle 0.001$ \\
\hline
\end{tabular}




\begin{tabular}{|c|c|c|c|c|c|c|}
\hline $\begin{array}{l}\text { Use of } \\
\text { antihypertensives, } \\
\text { n (\%) }\end{array}$ & $5239(49.7)$ & $1160(43.8)$ & $1240(46.8)$ & $1363(52.6)$ & $1476(55.7)$ & $\nabla 0.001$ \\
\hline $\begin{array}{l}\text { Use of } \\
\text { antilipidemic, n (\%) }\end{array}$ & 7787 (73.9) & $1703(64.3)$ & $1947(73.6)$ & $2026(78.2)$ & $2111(79.7)$ & $\nabla 0.001$ \\
\hline $\begin{array}{l}\text { CIMT, mm, median } \\
\text { (IQR) }\end{array}$ & $\begin{array}{l}0.10(0.09- \\
0.12)\end{array}$ & $\begin{array}{l}0.10(0.09- \\
0.12)\end{array}$ & $\begin{array}{l}0.10(0.09- \\
0.12)\end{array}$ & $\begin{array}{l}0.10(0.09- \\
0.12)\end{array}$ & $\begin{array}{l}0.10(0.09- \\
0.12)\end{array}$ & $\nabla 0.001$ \\
\hline $\begin{array}{l}\text { Carotid artery } \\
\text { plaque, } \mathrm{n}(\%)\end{array}$ & $\begin{array}{l}8079 \\
(76.69)\end{array}$ & $\begin{array}{l}1985 \\
(74.91)\end{array}$ & $\begin{array}{l}1996 \\
(75.41)\end{array}$ & $\begin{array}{l}2006 \\
(77.45)\end{array}$ & $\begin{array}{l}2092 \\
(79.00)\end{array}$ & $\nabla 0.001$ \\
\hline \multicolumn{6}{|c|}{ Glucose regulation state, $\mathrm{n}(\%)$} & $\varangle 0.001$ \\
\hline $\begin{array}{l}\text { Normal glucose } \\
\text { regulation }\end{array}$ & $3756(35.7)$ & $1735(65.5)$ & $1160(43.8)$ & $654(25.3)$ & $207(7.8)$ & \\
\hline Prediabetes & $2813(26.7)$ & $694(26.2)$ & $861(32.5)$ & $834(32.2)$ & $424(16.0)$ & \\
\hline Diabetes & $3966(37.6)$ & $221(8.3)$ & $626(23.7)$ & $1102(42.5)$ & $2017(76.2)$ & \\
\hline \multicolumn{6}{|c|}{ No. of carotid artery plaque, $n$ (\%) } & $\otimes 0.001$ \\
\hline 0 & $2456(23.3)$ & $665(25.1)$ & $651(24.6)$ & $584(22.5)$ & $556(21)$ & \\
\hline 1 & $406(3.9)$ & $96(3.6)$ & $100(3.8)$ & $100(3.9)$ & $110(4.2)$ & \\
\hline$\geq 2$ & $7673(72.8)$ & $1889(71.3)$ & $1896(71.6)$ & $1906(73.6)$ & $1982(74.8)$ & \\
\hline \multicolumn{6}{|c|}{ carotid artery plaque echo property, n (\%) } & $\otimes 0.001$ \\
\hline Hypoechoic plaque & $497(4.7)$ & $114(4.3)$ & $131(4.9)$ & $130(5)$ & $122(4.6)$ & \\
\hline Isoechoic plaque & $548(5.2)$ & $137(5.2)$ & $133(5)$ & $136(5.3)$ & $142(5.4)$ & \\
\hline $\begin{array}{l}\text { Hyperechoic } \\
\text { plaque }\end{array}$ & $4792(45.5)$ & $1211(45.7)$ & $1162(43.9)$ & $1194(46.1)$ & $1225(46.3)$ & \\
\hline Mixture plaque & $2242(21.3)$ & $523(19.7)$ & $570(21.5)$ & $546(21.1)$ & $603(22.8)$ & \\
\hline
\end{tabular}

Data are presented as median (interquartile) or number (proportion, \%); $P$-value was calculated by KruskalWallis test.

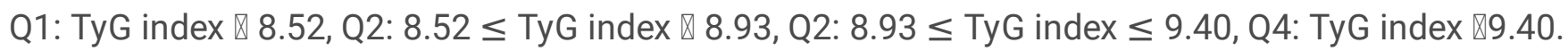

TyG: triglyceride-glucose index, SBP: Systolic blood pressure, DBP: Diastolic blood pressure, CRP: Creactionprotein, FPG: fasting plasma glucose, TC: Total cholesterol, TG: Triglycerides, HDL-C: High-density lipoprotein cholesterol, LDL-C: Low-density lipoprotein cholesterol, HbA1c: glycated haemoglobin, IQR: Interquartile rang

\section{Correlation between the TyG index and CAP}

As shown in Table 2, when Tyg index was used as a continuous variable, Multivariate logistic regression was shown that it was significantly correlated with the risk of CAP (OR: 1.31; 95\% Cl: 1.26-1.36). The correlation of 
the TyG index and the CAP occurrence was further explored when using the TyG index as a categorical variable. Multivariate logistic regression analysis showed that TyG index levels for Q3 and Q4 correlated with increased OR in CAP when Q1 as a reference, which the highest Q4 correlation of (OR: 1.42; 95\% Cl: 1.33 -1.53). In the unadjusted or adjusted model, the TyG index (quartiles) was consistent with the $P$ for trend of the CAP, when the TyG index was served as a continuous variable $(P<0.001$

\section{Table 2. Correlation between the TyG index and carotid artery plaques}

Variables Carotid artery plaques

\begin{tabular}{lllllll} 
& OR $(95 \% \mathrm{Cl})^{\mathrm{a}}$ & $P$-value & OR $(95 \% \mathrm{Cl})^{\mathrm{b}}$ & $P$-value & OR $(95 \% \mathrm{Cl})^{\mathrm{c}}$ & $P$-value \\
\hline TyG index & $1.14(1.11-1.17)$ & $<0.001$ & $1.28(1.24-1.31)$ & $<0.001$ & $1.31(1.26-1.36)$ & $<0.001$ \\
\hline Q1 & Reference & & Reference & & Reference & \\
\hline Q2 & $1.00(0.95-1.05)$ & 0.969 & $1.10(1.04-1.16)$ & 0.537 & $1.02(0.96-1.08)$ & 0.707 \\
\hline Q3 & $1.09(1.04-1.15)$ & 0.001 & $1.22(1.16-1.29)$ & 0.013 & $1.08(1.02-1.15)$ & 0.011 \\
\hline Q4 & $1.22(1.16-1.28)$ & $<0.001$ & $1.51(1.43-1.59)$ & $<0.001$ & $1.42(1.33-1.53)$ & $<0.001$ \\
\hline$P$-trend & & $<0.001$ & & $<0.001$ & & $<0.001$
\end{tabular}

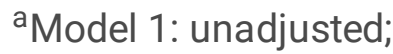

${ }^{\text {b} M o d e l ~ 2: ~ a d j u s t e d ~ f o r ~ s e x, ~ a g e, ~ S B P, ~ D B P ; ~}$

'Model 3: adjusted for sex, age, SBP, DBP, CRP, TC, HDL-C, LDL-C, smoking, drinking, hypertension, hyperlipidemia, use of antihypertensives, use of antilipidemic

Regardless of whether it is male or female, this relationship was still statistically significant after adjusting for variables. As shown in Table 3, after multivariate adjustment, the correlation between the Tyg index of female and CAP was (OR:1.38; 95\% Cl: 1.31-1.45), which was higher than that of male by (OR:1.23; 95\% Cl: 1.16, 1.30). As shown in Table 4, after multivariate adjustment, the TyG index of CHD patients is significantly correlated with CAP in different age. The OR value of middle-aged ( $\leq 60$ years old) patients (OR:1.14; 95\% Cl: 1.07-1.22) is

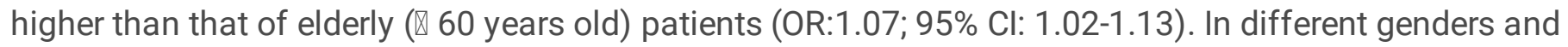
different age, Q3 and Q4 are significantly related to the increased risk of CAP, even after multivariate adjustmen, this relationship was still significant. Among the different genders and age, in the unadjusted or adjusted model, the TyG index (quartiles) was consistent with the $P$ for trend of the CAP, when the TyG index was served as a continuous variable $(P<0.001)$.

Table 3. Correlation between the TyG index and carotid artery plaques in different sex 
Sex Variables Carotid artery plaques

\begin{tabular}{|c|c|c|c|c|c|c|c|}
\hline & & OR $(95 \% \mathrm{Cl})^{a}$ & $P$-value & OR $(95 \% \mathrm{Cl})^{\mathrm{b}}$ & $P$-value & OR $(95 \% \mathrm{Cl})^{\mathrm{C}}$ & $P$-value \\
\hline \multirow[t]{6}{*}{ Male } & $\begin{array}{l}\text { TyG } \\
\text { index }\end{array}$ & $\begin{array}{l}0.94(0.91- \\
0.98)\end{array}$ & 0.003 & $\begin{array}{l}1.14(1.10- \\
1.19)\end{array}$ & $<0.001$ & $\begin{array}{l}1.23(1.16- \\
1.30)\end{array}$ & $<0.001$ \\
\hline & Q1 & Reference & & Reference & & Reference & \\
\hline & Q2 & $\begin{array}{l}1.00(0.91- \\
1.07)\end{array}$ & 0.789 & $\begin{array}{l}1.13(1.04- \\
1.23)\end{array}$ & 0.004 & $\begin{array}{l}1.07(0.97- \\
1.17)\end{array}$ & 0.178 \\
\hline & Q3 & $\begin{array}{l}0.95(0.88- \\
1.03)\end{array}$ & 0.213 & $\begin{array}{l}1.17(1.07- \\
1.28)\end{array}$ & $<0.001$ & $\begin{array}{l}1.05(0.95- \\
1.15)\end{array}$ & 0.371 \\
\hline & Q4 & $\begin{array}{l}0.87(0.80- \\
0.94)\end{array}$ & $<0.001$ & $\begin{array}{l}1.23(1.13- \\
1.34)\end{array}$ & $<0.001$ & $\begin{array}{l}1.23(1.11- \\
1.37)\end{array}$ & $<0.001$ \\
\hline & $P$-trend & & $<0.001$ & & $<0.001$ & & 0.001 \\
\hline \multirow[t]{6}{*}{ Female } & $\begin{array}{l}\text { TyG } \\
\text { index }\end{array}$ & $\begin{array}{l}1.34(1.30- \\
1.39)\end{array}$ & $<0.001$ & $\begin{array}{l}1.38(1.33- \\
1.44)\end{array}$ & $<0.001$ & $\begin{array}{l}1.38(1.31- \\
1.45)\end{array}$ & $<0.001$ \\
\hline & Q1 & Reference & & Reference & & Reference & \\
\hline & Q2 & $\begin{array}{l}1.08(1.01- \\
1.15)\end{array}$ & 0.019 & $\begin{array}{l}1.08(1.01- \\
1.16)\end{array}$ & 0.031 & $\begin{array}{l}0.99(0.92- \\
1.07)\end{array}$ & 0.849 \\
\hline & Q3 & $\begin{array}{l}1.31(1.23- \\
1.40)\end{array}$ & $<0.001$ & $\begin{array}{l}1.25 \text { (1.17- } \\
1.34)\end{array}$ & $<0.001$ & $\begin{array}{l}1.10(1.02- \\
1.19)\end{array}$ & 0.019 \\
\hline & Q4 & $\begin{array}{l}1.62(1.52- \\
1.74)\end{array}$ & $<0.001$ & $\begin{array}{l}1.74(1.61- \\
1.87)\end{array}$ & $<0.001$ & $\begin{array}{l}1.57(1.44- \\
1.72)\end{array}$ & $<0.001$ \\
\hline & $P$-trend & & $<0.001$ & & $<0.001$ & & $<0.001$ \\
\hline
\end{tabular}

a Model 1: unadjusted;

bModel 2: adjusted for age, SBP, DBP;

'Model 3: adjusted for age, SBP, DBP, CRP, TC, HDL-C, LDL-C, smoking, drinking, Hypertension, Hyperlipidemia, Use of antihypertensives, Use of antilipidemic.

Table 4. Correlation between the TyG index and carotid artery plaques in different age 
Age Variables Carotid artery plaques

\begin{tabular}{|c|c|c|c|c|c|c|c|}
\hline & & OR $(95 \% \mathrm{Cl})^{\mathrm{a}}$ & $P$-value & OR $(95 \% \mathrm{Cl})^{\mathrm{b}}$ & $P$-value & OR $(95 \% \mathrm{Cl})^{\mathrm{C}}$ & $P$-value \\
\hline \multirow[t]{6}{*}{$\leq 60$} & TyG index & $1.37(1.32-1.42)$ & $\otimes 0.001$ & $1.27(1.22-1.32)$ & $\otimes 0.001$ & $1.14(1.07-1.22)$ & $\triangle 0.001$ \\
\hline & Q1 & Reference & & Reference & & Reference & \\
\hline & Q2 & $1.19(1.09-1.29)$ & $\otimes 0.001$ & $1.18(1.08-1.30)$ & $\otimes 0.001$ & $1.04(0.94-1.16)$ & 0.400 \\
\hline & Q3 & $1.59(1.45-1.73)$ & $\otimes 0.001$ & $1.45(1.32-1.59)$ & $\otimes 0.001$ & $1.17(1.05-1.30)$ & 0.010 \\
\hline & Q4 & $1.90(1.75-2.06)$ & $\otimes 0.001$ & $1.68(1.54-1.83)$ & $\llbracket 0.001$ & $1.24(1.10-1.39)$ & $\otimes 0.001$ \\
\hline & $P$-trend & & $\otimes 0.001$ & & $\llbracket 0.001$ & & $\triangle 0.001$ \\
\hline \multirow[t]{6}{*}{$₫ 60$} & TyG index & $1.16(1.12-1.21)$ & $\otimes 0.001$ & $1.21(1.16-1.25)$ & $\otimes 0.001$ & $1.07(1.02-1.13)$ & 0.010 \\
\hline & Q1 & Reference & & Reference & & Reference & \\
\hline & Q2 & $0.99(0.93-1.06)$ & 0.840 & $1.05(1.00-1.12)$ & 0.170 & $0.95(0.88-1.02)$ & 0.150 \\
\hline & Q3 & $0.99(0.93-1.05)$ & 0.720 & $1.07(1.00-1.15)$ & 0.040 & $0.89(0.83-0.96)$ & $₫ 0.001$ \\
\hline & Q4 & $1.23(1.15-1.32)$ & $\otimes 0.001$ & $1.30(1.21-1.39)$ & $\llbracket 0.001$ & $1.02(0.93-1.11)$ & 0.760 \\
\hline & $P$-trend & & $\otimes 0.001$ & & $\otimes 0.001$ & & $\otimes 0.001$ \\
\hline
\end{tabular}

${ }^{a}$ Model 1: unadjusted;

${ }^{\mathrm{b}}$ Model 2: adjusted for sex, SBP, DBP;

'Model 3: adjusted for sex, SBP, DBP, CRP, TC, HDL-C, LDL-C, smoking, drinking, hypertension, hyperlipidemia, use of antihypertensives, use of antilipidemic

\section{Correlation between the TyG index and CAP in different glucose regulation state}

As shown in Table 5, under different glucose metabolism states, after multivariate adjustmen, TyG index in CHD patients had a significant correlation with the risk of CAP, and the OR value of DM is the highest (OR:1.35; $95 \%$ Cl:1.26-1.45). Taking the Q1 as a reference, Q3, Q4 and DM were significantly correlation with the increased risk of CAP during normal glucose tolerance (NGT). In the unadjusted or adjusted model, the TyG index (quartiles) was consistent with the $P$ for trend of the CAP, when the TyG index was served as a continuous variable ( $P$ $<0.001)$.

There was a significant correlation between TyG index and CAP risk in male patients under NGT (OR:1.32; 95\% 1.16-1.51), and the correlation between females (OR:1.45; $95 \% \mathrm{Cl}: 1.32-1.59)$ under DM status was higher than males (OR:1.17; 95\% Cl:1.05-1.30). (Table 6) In CHD patients ( $\leq 60$ years old), TyG index was significantly associated with CAP in NGT patients (OR:1.23; 95\% Cl:1.07-1.40). TyG index was significantly associated with the risk of CAP in DM patients (>60 years old) (OR:1.18; $95 \%$ Cl:1.07-1.30). (Table 7) 
Glucose regulation Variables Carotid artery plaques state

$\begin{array}{llllll}\mathrm{OR}(95 \% & P \text { - } & \mathrm{OR}(95 \% & P \text {-value } & \mathrm{OR}(95 \% & \begin{array}{l}P \text { - } \\ \mathrm{Cl})^{\mathrm{a}}\end{array} \\ \text { value } & \mathrm{Cl})^{\mathrm{b}} & & \mathrm{Cl})^{\mathrm{C}} & \text { value }\end{array}$

\begin{tabular}{|c|c|c|c|c|c|c|c|}
\hline \multirow[t]{6}{*}{$\begin{array}{l}\text { Normal glucose } \\
\text { regulation }\end{array}$} & $\begin{array}{l}\text { TyG } \\
\text { index }\end{array}$ & $\begin{array}{l}0.95(0.90- \\
1.01)\end{array}$ & 0.080 & $\begin{array}{l}1.16(1.09- \\
1.24)\end{array}$ & $<0.001$ & $\begin{array}{l}1.16(1.07- \\
1.27)\end{array}$ & 0.001 \\
\hline & Q1 & Reference & & Reference & & Reference & \\
\hline & Q2 & $\begin{array}{l}0.98(0.92- \\
1.05)\end{array}$ & 0.526 & $\begin{array}{l}1.11(1.03- \\
1.19)\end{array}$ & 0.007 & $\begin{array}{l}1.04(0.95- \\
1.12)\end{array}$ & 0.419 \\
\hline & Q3 & $\begin{array}{l}0.94(0.86- \\
1.02)\end{array}$ & 0.112 & $\begin{array}{l}1.18(1.08- \\
1.29)\end{array}$ & $<0.001$ & $\begin{array}{l}1.09(0.98- \\
1.21)\end{array}$ & 0.109 \\
\hline & Q4 & $\begin{array}{l}0.81(0.71- \\
0.92)\end{array}$ & 0.001 & $\begin{array}{l}1.11(0.97- \\
1.28)\end{array}$ & 0.129 & $\begin{array}{l}1.22(1.03- \\
1.45)\end{array}$ & 0.024 \\
\hline & $P$-trend & & $<.001$ & & $<0.001$ & & $<.001$ \\
\hline
\end{tabular}

\begin{tabular}{|c|c|c|c|c|c|c|c|}
\hline \multirow[t]{6}{*}{ Prediabetes } & $\begin{array}{l}\text { TyG } \\
\text { index }\end{array}$ & $\begin{array}{l}0.87(0.82- \\
0.92)\end{array}$ & $\begin{array}{l}< \\
0.001\end{array}$ & $\begin{array}{l}1.06(0.99- \\
1.14)\end{array}$ & 0.078 & $\begin{array}{l}1.11(1.01- \\
1.23)\end{array}$ & 0.036 \\
\hline & Q1 & Reference & & Reference & & Reference & \\
\hline & Q2 & $\begin{array}{l}0.90(0.83- \\
0.99)\end{array}$ & 0.032 & $\begin{array}{l}1.06(0.96- \\
1.17)\end{array}$ & 0.288 & $\begin{array}{l}0.94(0.84- \\
1.05)\end{array}$ & 0.250 \\
\hline & Q3 & $\begin{array}{l}1.02(0.93- \\
1.12)\end{array}$ & 0.645 & $\begin{array}{l}1.23(1.11- \\
1.37)\end{array}$ & $<0.001$ & $\begin{array}{l}1.08(0.96- \\
1.22)\end{array}$ & 0.192 \\
\hline & Q4 & $\begin{array}{l}0.69(0.62- \\
0.77)\end{array}$ & $\begin{array}{l}< \\
0.001\end{array}$ & $\begin{array}{l}0.99(0.88- \\
1.12)\end{array}$ & 0.879 & $\begin{array}{l}0.97(0.83- \\
1.14)\end{array}$ & 0.721 \\
\hline & $P$-trend & & $\begin{array}{l}< \\
0.001\end{array}$ & & $<0.001$ & & $\begin{array}{l}<.001 \\
0.00\end{array}$ \\
\hline \multirow[t]{6}{*}{ Diabetes } & $\begin{array}{l}\text { TyG } \\
\text { index }\end{array}$ & $\begin{array}{l}1.16(1.11- \\
1.22)\end{array}$ & $\begin{array}{l}< \\
0.001\end{array}$ & $\begin{array}{l}1.36(1.30- \\
1.43)\end{array}$ & $<0.001$ & $\begin{array}{l}1.35(1.26- \\
1.45)\end{array}$ & $\begin{array}{l}< \\
0.001\end{array}$ \\
\hline & Q1 & Reference & & Reference & & Reference & \\
\hline & Q2 & $\begin{array}{l}1.11(0.97- \\
1.28)\end{array}$ & 0.142 & $\begin{array}{l}1.26(1.09- \\
1.47)\end{array}$ & 0.003 & $\begin{array}{l}1.13(0.96- \\
1.33)\end{array}$ & 0.147 \\
\hline & Q3 & $\begin{array}{l}1.18(1.03- \\
1.35)\end{array}$ & 0.017 & $\begin{array}{l}1.37(1.19- \\
1.58)\end{array}$ & $<0.001$ & $\begin{array}{l}1.07(0.92- \\
1.25)\end{array}$ & 0.398 \\
\hline & Q4 & $\begin{array}{l}1.39(1.22- \\
1.58)\end{array}$ & $<.001$ & $\begin{array}{l}1.87(1.63- \\
2.15)\end{array}$ & $<0.001$ & $\begin{array}{l}1.41(1.20- \\
1.66)\end{array}$ & $\begin{array}{l}<.001 \\
0.001\end{array}$ \\
\hline & $P$-trend & & $<.001$ & & $<0.001$ & & $\begin{array}{l}< \\
0.001\end{array}$ \\
\hline
\end{tabular}

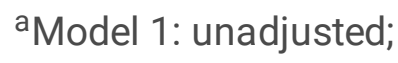

bModel 2: adjusted for age, sex, SBP, DBP; 
'Model 3: adjusted for age, sex, SBP, DBP, CRP, TC, HDL-C, LDL-C, smoking, drinking, hypertension, hyperlipidemia, use of antihypertensives, use of antilipidemic.

Table 6. Correlation between the TyG index and carotid artery plaques in different glucose regulation state and $\operatorname{sex}$ 


\begin{tabular}{|c|c|c|c|c|c|c|c|c|}
\hline \multirow[t]{2}{*}{ Sex } & \multirow{2}{*}{$\begin{array}{l}\text { Glucose } \\
\text { regulation } \\
\text { state }\end{array}$} & \multirow[t]{2}{*}{ Variables } & \multicolumn{6}{|c|}{ Carotid artery plaques } \\
\hline & & & $\begin{array}{l}\text { OR }(95 \% \\
\mathrm{Cl})^{\mathrm{a}}\end{array}$ & $P$-value & $\begin{array}{l}\text { OR }(95 \% \\
\mathrm{Cl})^{\mathrm{b}}\end{array}$ & $P$-value & $\begin{array}{l}\text { OR }(95 \% \\
\mathrm{Cl})^{\mathrm{C}}\end{array}$ & $P$-value \\
\hline \multirow[t]{17}{*}{ Male } & \multirow[t]{6}{*}{$\begin{array}{l}\text { Normal } \\
\text { glucose } \\
\text { regulation }\end{array}$} & $\begin{array}{l}\text { TyG } \\
\text { index }\end{array}$ & $\begin{array}{l}0.88 \\
(0.81- \\
0.96)\end{array}$ & 0.005 & $\begin{array}{l}1.24 \\
(1.13- \\
1.36)\end{array}$ & $<0.001$ & $\begin{array}{l}1.32 \\
(1.16- \\
1.51)\end{array}$ & $<0.001$ \\
\hline & & Q1 & Reference & & Reference & & Reference & \\
\hline & & Q2 & $\begin{array}{l}0.98 \\
(0.88- \\
1.10)\end{array}$ & 0.751 & $\begin{array}{l}1.23 \\
(1.09- \\
1.38)\end{array}$ & 0.001 & $\begin{array}{l}1.11 \\
(0.97- \\
1.27)\end{array}$ & 0.124 \\
\hline & & Q3 & $\begin{array}{l}0.79 \\
(0.69- \\
0.90)\end{array}$ & $<0.001$ & $\begin{array}{l}1.10 \\
(0.96- \\
1.27)\end{array}$ & 0.182 & $\begin{array}{l}0.89 \\
(0.75- \\
1.06)\end{array}$ & 0.177 \\
\hline & & Q4 & $\begin{array}{l}0.57 \\
(0.48- \\
0.68)\end{array}$ & $<0.001$ & $\begin{array}{l}1.00 \\
(0.82- \\
1.22)\end{array}$ & 0.974 & $\begin{array}{l}1.17 \\
(0.89- \\
1.54)\end{array}$ & 0.255 \\
\hline & & $P$-trend & & $<0.001$ & & 0.275 & & 0.980 \\
\hline & \multirow[t]{6}{*}{ Prediabetes } & $\begin{array}{l}\text { TyG } \\
\text { index }\end{array}$ & $\begin{array}{l}0.67 \\
(0.61- \\
0.74)\end{array}$ & $<0.001$ & $\begin{array}{l}0.97 \\
(0.87- \\
1.07)\end{array}$ & 0.505 & $\begin{array}{l}1.01 \\
(0.87- \\
1.19)\end{array}$ & 0.859 \\
\hline & & Q1 & Reference & & Reference & & Reference & \\
\hline & & Q2 & $\begin{array}{l}0.94 \\
(0.81- \\
1.10)\end{array}$ & 0.420 & $\begin{array}{l}1.05 \\
(0.89- \\
1.24)\end{array}$ & 0.552 & $\begin{array}{l}0.90 \\
(0.75- \\
1.07)\end{array}$ & 0.221 \\
\hline & & Q3 & $\begin{array}{l}0.85 \\
(0.73- \\
0.99)\end{array}$ & 0.038 & $\begin{array}{l}1.18 \\
(1.00- \\
1.39)\end{array}$ & 0.055 & $\begin{array}{l}1.03 \\
(0.85- \\
1.24)\end{array}$ & 0.799 \\
\hline & & Q4 & $\begin{array}{l}0.49 \\
(0.42- \\
0.58)\end{array}$ & $<0.001$ & $\begin{array}{l}0.83 \\
(0.69- \\
1.00)\end{array}$ & 0.045 & $\begin{array}{l}0.72 \\
(0.56- \\
0.93)\end{array}$ & 0.012 \\
\hline & & $P$-trend & & 0.393 & & 0.278 & & 0.148 \\
\hline & \multirow[t]{5}{*}{ Diabetes } & $\begin{array}{l}\text { TyG } \\
\text { index }\end{array}$ & $\begin{array}{l}0.91 \\
(0.84- \\
0.97)\end{array}$ & 0.005 & $\begin{array}{l}1.11 \\
(1.03- \\
1.19)\end{array}$ & 0.009 & $\begin{array}{l}1.17 \\
(1.05- \\
1.30)\end{array}$ & 0.004 \\
\hline & & Q1 & Reference & & Reference & & Reference & \\
\hline & & Q2 & $\begin{array}{l}0.99 \\
(0.78- \\
1.24)\end{array}$ & 0.904 & $\begin{array}{l}1.19 \\
(0.94- \\
1.51)\end{array}$ & 0.143 & $\begin{array}{l}1.09 \\
(0.85- \\
1.40)\end{array}$ & 0.488 \\
\hline & & Q3 & $\begin{array}{l}1.06 \\
(0.85- \\
1.32)\end{array}$ & 0.592 & $\begin{array}{l}1.34 \\
(1.07- \\
1.67)\end{array}$ & 0.011 & $\begin{array}{l}1.08 \\
(0.85- \\
1.36)\end{array}$ & 0.549 \\
\hline & & Q4 & $\begin{array}{l}0.98 \\
(0.79-\end{array}$ & 0.821 & $\begin{array}{l}1.48 \\
(1.19-\end{array}$ & $<0.001$ & $\begin{array}{l}1.25 \\
(0.98-\end{array}$ & 0.071 \\
\hline
\end{tabular}

Page 13/25 


\begin{tabular}{|c|c|c|c|c|c|c|c|c|}
\hline & & & $1.20)$ & & 1.84) & & 1.59) & \\
\hline & & $P$-trend & & 0.662 & & $<0.001$ & & 0.032 \\
\hline Female & $\begin{array}{l}\text { Normal } \\
\text { glucose } \\
\text { regulation }\end{array}$ & $\begin{array}{l}\text { TyG } \\
\text { index }\end{array}$ & $\begin{array}{l}1.05 \\
(0.97- \\
1.14)\end{array}$ & 0.198 & $\begin{array}{l}1.09 \\
(1.00- \\
1.19)\end{array}$ & 0.046 & $\begin{array}{l}1.07 \\
(0.95- \\
1.21)\end{array}$ & 0.262 \\
\hline & & Q1 & Reference & & Reference & & Reference & \\
\hline & & Q2 & $\begin{array}{l}1.06 \\
(0.97- \\
1.15)\end{array}$ & 0.223 & $\begin{array}{l}1.04 \\
(0.94- \\
1.14)\end{array}$ & 0.469 & $\begin{array}{l}1.01 \\
(0.90- \\
1.12)\end{array}$ & 0.904 \\
\hline & & Q3 & $\begin{array}{l}1.17 \\
(1.06- \\
1.30)\end{array}$ & 0.003 & $\begin{array}{l}1.22 \\
(1.09- \\
1.37)\end{array}$ & 0.001 & $\begin{array}{l}1.24 \\
(1.08- \\
1.42)\end{array}$ & 0.003 \\
\hline & & Q4 & $\begin{array}{l}1.03 \\
(0.86- \\
1.24)\end{array}$ & 0.739 & $\begin{array}{l}1.23 \\
(1.01-1.5)\end{array}$ & 0.041 & $\begin{array}{l}1.35 \\
(1.06- \\
1.72)\end{array}$ & 0.014 \\
\hline & & $P$-trend & & 0.019 & & $<0.001$ & & 0.002 \\
\hline & Prediabetes & $\begin{array}{l}\text { TyG } \\
\text { index }\end{array}$ & $\begin{array}{l}1.05 \\
(0.97- \\
1.14)\end{array}$ & 0.244 & $\begin{array}{l}1.14 \\
(1.04- \\
1.24)\end{array}$ & 0.005 & $\begin{array}{l}1.21 \\
(1.06- \\
1.39)\end{array}$ & 0.005 \\
\hline & & Q1 & Reference & & Reference & & Reference & \\
\hline & & Q2 & $\begin{array}{l}0.94 \\
(0.84- \\
1.06)\end{array}$ & 0.327 & $\begin{array}{l}1.05 \\
(0.93- \\
1.20)\end{array}$ & 0.410 & $\begin{array}{l}0.94 \\
(0.81- \\
1.07)\end{array}$ & 0.341 \\
\hline & & Q3 & $\begin{array}{l}1.22 \\
(1.08- \\
1.38)\end{array}$ & 0.001 & $\begin{array}{l}1.26 \\
(1.11- \\
1.44)\end{array}$ & $<0.001$ & $\begin{array}{l}1.12 \\
(0.96- \\
1.31)\end{array}$ & 0.153 \\
\hline & & Q4 & $\begin{array}{l}0.89 \\
(0.77- \\
1.03)\end{array}$ & 0.127 & $\begin{array}{l}1.12 \\
(0.96- \\
1.31)\end{array}$ & 0.145 & $\begin{array}{l}1.20 \\
(0.97- \\
1.49)\end{array}$ & 0.098 \\
\hline & & $P$-trend & & 0.407 & & 0.005 & & 0.033 \\
\hline & Diabetes & $\begin{array}{l}\text { TyG } \\
\text { index }\end{array}$ & $\begin{array}{l}1.44 \\
(1.35- \\
1.53)\end{array}$ & $<0.001$ & $\begin{array}{l}1.58 \\
(1.48- \\
1.69)\end{array}$ & $<0.001$ & $\begin{array}{l}1.45 \\
(1.32- \\
1.59)\end{array}$ & $<0.001$ \\
\hline & & Q1 & Reference & & Reference & & Reference & \\
\hline & & Q2 & $\begin{array}{l}1.31 \\
(1.08- \\
1.59)\end{array}$ & 0.005 & $\begin{array}{l}1.30 \\
(1.06- \\
1.59)\end{array}$ & 0.012 & $\begin{array}{l}1.11 \\
(0.89- \\
1.39)\end{array}$ & 0.340 \\
\hline & & Q3 & $\begin{array}{l}1.44 \\
(1.21- \\
1.73)\end{array}$ & $<0.001$ & $\begin{array}{l}1.38 \\
(1.14- \\
1.67)\end{array}$ & 0.001 & $\begin{array}{l}0.98 \\
(0.79- \\
1.21)\end{array}$ & 0.850 \\
\hline & & Q4 & $\begin{array}{l}1.97 \\
(1.65- \\
2.34)\end{array}$ & $<0.001$ & $\begin{array}{l}2.15 \\
(1.78- \\
2.58)\end{array}$ & $<0.001$ & $\begin{array}{l}1.39 \\
(1.12- \\
1.73)\end{array}$ & 0.003 \\
\hline
\end{tabular}


aModel 1: unadjusted;

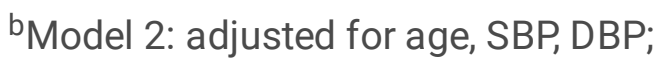

CModel 3: adjusted for age, SBP, DBP, CRP, TC, HDL-C, LDL-C, smoking, drinking, hypertension, hyperlipidemia, use of antihypertensives, use of antilipidemic.

Table 7. Correlation between the TyG index and carotid artery plaques in different different glucose regulation state and age 


\begin{tabular}{|c|c|c|c|c|c|c|c|c|}
\hline \multirow[t]{2}{*}{ Age } & \multirow{2}{*}{$\begin{array}{l}\text { Glucose } \\
\text { regulation } \\
\text { state }\end{array}$} & \multirow[t]{2}{*}{ Variables } & \multicolumn{6}{|c|}{ carotid artery plaques } \\
\hline & & & $\begin{array}{l}\text { OR }(95 \% \\
\mathrm{Cl})^{\mathrm{a}}\end{array}$ & $P$-value & $\begin{array}{l}\text { OR }(95 \% \\
\mathrm{Cl})^{\mathrm{b}}\end{array}$ & $P$-value & $\begin{array}{l}\text { OR }(95 \% \\
\mathrm{Cl})^{\mathrm{c}}\end{array}$ & $P$-value \\
\hline \multirow[t]{17}{*}{$\leq 60$} & \multirow[t]{6}{*}{$\begin{array}{l}\text { Normal } \\
\text { glucose } \\
\text { regulation }\end{array}$} & $\begin{array}{l}\text { TyG } \\
\text { index }\end{array}$ & $\begin{array}{l}1.32 \\
(1.22- \\
1.44)\end{array}$ & $\varangle 0.001$ & $\begin{array}{l}1.16 \\
(1.06- \\
1.28)\end{array}$ & $\llbracket 0.001$ & $\begin{array}{l}1.23 \\
(1.07- \\
1.40)\end{array}$ & $\varangle 0.001$ \\
\hline & & Q1 & Reference & & Reference & & Reference & \\
\hline & & Q2 & $\begin{array}{l}1.27 \\
(1.14- \\
1.43)\end{array}$ & $\otimes 0.001$ & $\begin{array}{l}1.24 \\
(1.10- \\
1.40)\end{array}$ & $\llbracket 0.001$ & $\begin{array}{l}1.22 \\
(1.06- \\
1.40)\end{array}$ & $\varangle 0.001$ \\
\hline & & Q3 & $\begin{array}{l}1.53 \\
(1.34- \\
1.74)\end{array}$ & $\otimes 0.001$ & $\begin{array}{l}1.40 \\
(1.22- \\
1.60)\end{array}$ & $\llbracket 0.001$ & $\begin{array}{l}1.32 \\
(1.12- \\
1.57)\end{array}$ & $\llbracket 0.001$ \\
\hline & & Q4 & $\begin{array}{l}1.38 \\
(1.15- \\
1.64)\end{array}$ & $\varangle 0.001$ & $\begin{array}{l}1.09 \\
(0.91- \\
1.32)\end{array}$ & 0.360 & $\begin{array}{l}1.26 \\
(0.98- \\
1.63)\end{array}$ & 0.070 \\
\hline & & $P$-trend & & $\otimes 0.001$ & & $\llbracket 0.001$ & & $\varangle 0.001$ \\
\hline & \multirow[t]{6}{*}{ Prediabetes } & $\begin{array}{l}\text { TyG } \\
\text { index }\end{array}$ & $\begin{array}{l}1.05 \\
(0.96- \\
1.15)\end{array}$ & 0.290 & $\begin{array}{l}0.99 \\
(0.90- \\
1.09)\end{array}$ & 0.770 & $\begin{array}{l}1.03 \\
(0.88- \\
1.21)\end{array}$ & 0.710 \\
\hline & & Q1 & Reference & & Reference & & Reference & \\
\hline & & Q2 & $\begin{array}{l}0.86 \\
(0.73- \\
1.02)\end{array}$ & 0.090 & $\begin{array}{l}0.92 \\
(0.77- \\
1.09)\end{array}$ & 0.320 & $\begin{array}{l}0.73 \\
(0.60- \\
0.89)\end{array}$ & $\varangle 0.001$ \\
\hline & & Q3 & $\begin{array}{l}1.21 \\
(1.02- \\
1.43)\end{array}$ & 0.030 & $\begin{array}{l}1.12 \\
(0.94- \\
1.34)\end{array}$ & 0.190 & $\begin{array}{l}0.88 \\
(0.71- \\
1.09)\end{array}$ & 0.230 \\
\hline & & Q4 & $\begin{array}{l}1.08 \\
(0.91- \\
1.29)\end{array}$ & 0.390 & $\begin{array}{l}1.04 \\
(0.87- \\
1.26)\end{array}$ & 0.650 & $\begin{array}{l}0.99 \\
(0.76- \\
1.28)\end{array}$ & 0.930 \\
\hline & & $P$-trend & & 0.014 & & 0.015 & & 0.148 \\
\hline & \multirow[t]{5}{*}{ Diabetes } & $\begin{array}{l}\text { TyG } \\
\text { index }\end{array}$ & $\begin{array}{l}1.21 \\
(1.13- \\
1.30)\end{array}$ & $\otimes 0.001$ & $\begin{array}{l}1.16 \\
(1.08- \\
1.25)\end{array}$ & $\llbracket 0.001$ & $\begin{array}{l}0.99 \\
(0.88- \\
1.12)\end{array}$ & 0.860 \\
\hline & & Q1 & Reference & & Reference & & Reference & \\
\hline & & Q2 & $\begin{array}{l}1.32 \\
(0.98- \\
1.78)\end{array}$ & 0.070 & $\begin{array}{l}1.23 \\
(0.91- \\
1.67)\end{array}$ & 0.190 & $\begin{array}{l}0.88 \\
(0.63- \\
1.25)\end{array}$ & 0.480 \\
\hline & & Q3 & $\begin{array}{l}1.71 \\
(1.29- \\
2.27)\end{array}$ & $\otimes 0.001$ & $\begin{array}{l}1.49 \\
(1.11- \\
2.00)\end{array}$ & 0.010 & $\begin{array}{l}0.88 \\
(0.63- \\
1.23)\end{array}$ & 0.470 \\
\hline & & Q4 & $\begin{array}{l}1.98 \\
(1.51- \\
2.60)\end{array}$ & $\otimes 0.001$ & $\begin{array}{l}1.69 \\
(1.27- \\
2.24)\end{array}$ & $\llbracket 0.001$ & $\begin{array}{l}0.84 \\
(0.60- \\
1.17)\end{array}$ & 0.310 \\
\hline
\end{tabular}




\begin{tabular}{|c|c|c|c|c|c|c|c|c|}
\hline & & $P$-trend & & $\nabla 0.001$ & & $\bowtie 0.001$ & & 0.076 \\
\hline \multirow{6}{*}{\multicolumn{2}{|c|}{$\begin{array}{l}\text { Normal } \\
\text { glucose } \\
\text { regulation }\end{array}$}} & $\begin{array}{l}\text { TyG } \\
\text { index }\end{array}$ & $\begin{array}{l}0.94 \\
(0.87- \\
1.02)\end{array}$ & 0.160 & $\begin{array}{l}1.05 \\
(0.96- \\
1.14)\end{array}$ & 0.300 & $\begin{array}{l}1.01 \\
(0.90- \\
1.14)\end{array}$ & 0.840 \\
\hline & & Q1 & Reference & & Reference & & Reference & \\
\hline & & Q2 & $\begin{array}{l}0.94 \\
(0.86- \\
1.03)\end{array}$ & 0.200 & $\begin{array}{l}1.01 \\
(0.92- \\
1.11)\end{array}$ & 0.780 & $\begin{array}{l}0.94 \\
(0.85- \\
1.04)\end{array}$ & 0.240 \\
\hline & & Q3 & $\begin{array}{l}0.85 \\
(0.76- \\
0.95)\end{array}$ & $\varangle 0.001$ & $\begin{array}{l}0.99 \\
(0.88- \\
1.11)\end{array}$ & 0.890 & $\begin{array}{l}0.90 \\
(0.78- \\
1.03)\end{array}$ & 0.120 \\
\hline & & Q4 & $\begin{array}{l}0.90 \\
(0.74- \\
1.10)\end{array}$ & 0.320 & $\begin{array}{l}0.95 \\
(0.77- \\
1.17)\end{array}$ & 0.640 & $\begin{array}{l}1.05 \\
(0.82- \\
1.35)\end{array}$ & 0.700 \\
\hline & & $P$-trend & & 0.007 & & 0.269 & & 0.906 \\
\hline & \multirow[t]{6}{*}{ Prediabetes } & $\begin{array}{l}\text { TyG } \\
\text { index }\end{array}$ & $\begin{array}{l}0.98 \\
(0.90- \\
1.07)\end{array}$ & 0.640 & $\begin{array}{l}1.03 \\
(0.94- \\
1.13)\end{array}$ & 0.510 & $\begin{array}{l}1.01 \\
(0.89- \\
1.15)\end{array}$ & 0.880 \\
\hline & & Q1 & Reference & & Reference & & Reference & \\
\hline & & Q2 & $\begin{array}{l}1.03 \\
(0.91- \\
1.15)\end{array}$ & 0.680 & $\begin{array}{l}1.08 \\
(0.96- \\
1.21)\end{array}$ & 0.230 & $\begin{array}{l}0.96 \\
(0.84- \\
1.09)\end{array}$ & 0.500 \\
\hline & & Q3 & $\begin{array}{l}1.09 \\
(0.97- \\
1.23)\end{array}$ & 0.150 & $\begin{array}{l}1.19 \\
(1.05- \\
1.35)\end{array}$ & 0.010 & $\begin{array}{l}1.03 \\
(0.89- \\
1.19)\end{array}$ & 0.710 \\
\hline & & Q4 & $\begin{array}{l}0.76 \\
(0.66- \\
0.88)\end{array}$ & $\nabla 0.001$ & $\begin{array}{l}0.80 \\
(0.69- \\
0.94)\end{array}$ & 0.010 & $\begin{array}{l}0.74 \\
(0.60- \\
0.91)\end{array}$ & 0.010 \\
\hline & & $P$-trend & & 0.046 & & 0.935 & & 0.685 \\
\hline & \multirow[t]{6}{*}{ Diabetes } & $\begin{array}{l}\text { TyG } \\
\text { index }\end{array}$ & $\begin{array}{l}1.38 \\
(1.29- \\
1.47)\end{array}$ & $\varangle 0.001$ & $\begin{array}{l}1.43 \\
(1.34- \\
1.53)\end{array}$ & 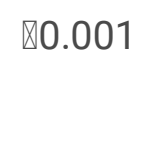 & $\begin{array}{l}1.18 \\
(1.07- \\
1.30)\end{array}$ & $\varangle 0.001$ \\
\hline & & Q1 & Reference & & Reference & & Reference & \\
\hline & & Q2 & $\begin{array}{l}1.18 \\
(0.99- \\
1.40)\end{array}$ & 0.060 & $\begin{array}{l}1.25 \\
(1.05- \\
1.49)\end{array}$ & 0.010 & $\begin{array}{l}1.13 \\
(0.94- \\
1.35)\end{array}$ & 0.210 \\
\hline & & Q3 & $\begin{array}{l}1.15 \\
(0.98- \\
1.35)\end{array}$ & 0.080 & $\begin{array}{l}1.25 \\
(1.06- \\
1.47)\end{array}$ & 0.010 & $\begin{array}{l}0.94 \\
(0.79- \\
1.13)\end{array}$ & 0.510 \\
\hline & & Q4 & $\begin{array}{l}1.63 \\
(1.39- \\
1.90)\end{array}$ & $\bowtie 0.001$ & $\begin{array}{l}1.78 \\
(1.52- \\
2.09)\end{array}$ & $\llbracket 0.001$ & $\begin{array}{l}1.18 \\
(0.98- \\
1.42)\end{array}$ & 0.090 \\
\hline & & $P$-trend & & $\nabla 0.001$ & & $\nabla 0.001$ & & $\varangle 0.001$ \\
\hline
\end{tabular}


aModel 1: unadjusted;

${ }^{b}$ Model 2: adjusted for sex, SBP, DBP;

CModel 3: adjusted for sex, SBP, DBP, CRP, TC, HDL-C, LDL-C, smoking, drinking, hypertension, hyperlipidemia, use of antihypertensives, use of antilipidemic.

\section{Discussion}

In this study, we determined a significant correlation between TyG index and CAP occurrence. This is the first large-scale study to prove the relationship between TyG index and CAP in CHD patients, and further study the relationship between TyG index and CAP in CHD patients under different genders, different ages and different glucose metabolism states.

A number of research reports in recent years had shown that TyG index is closely related to the homeostasis model assessment of insulin resistance (HOMA-IR) [27]. And the predictive value of TyG index for IR is better than HOMA-IR [28]. Therefore, the TyG index may better reflect the indicator of peripheral IR. In a cross-sectional study, TyG index was positively correlated with the prevalence of CAD and can be used as a marker of AS [29]. Compared with patients with the lowest TyG index, patients in the quartile with the highest TyG index have a higher risk of stroke and myocardial infarction (MI) [30]. TyG index were significantly correlated with the progression of arterial stiffness in hypertensive people but not in prehypertensive people [31]. Many studies show that TyG index was closely related to coronary artery calcification and carotid AS [32]. This is consistent with the results of this study. The related mechanism of action may have many aspects. Firstly, insulin can cause lipid hyaluronic degeneration by enhancing sympathetic nerve activity or acting as a growth factor. Lipohyaline deposition can block small arteries, leading to the development of CVD [33]. Secondly, Tyg index is related to inflammation. IR can induce inflammation, oxidative stress and metabolic changes to cause damage to the vascular endothelium due to inflammation [34]. Therefore, the TyG index in CHD patients was associated with the occurrence of CAP, and a high level of Tyg index was associated with the occurrence of higher CAP.

The close association between the TyG index and cSVD may be caused by other concomitant metabolic syndromes. IR patients usually have other comorbidities, such as hypertension, DM and obesity [35-36]. TyG index has received attention in the field of DM and metabolism, and has a positive impact on the assessment and prediction of IR and metabolic syndrome in DM patients. A higher TyG index was associated with an increased risk of coronary artery stenosis in asymptomatic T2DM patients [37]. But there is cardiometabolic heterogeneity in non-DM individuals [38-39]. Consistent with the results of this study, this study found that by observing different glucose metabolism states and adjusting the confounding variables, there is a significant correlation between TyG in CHD patients and the risk of CAP, and the OR value is the highest in the state of DM.

Recent studies have shown that female have a lower risk of cardiovascular disease than male, but hyperglycemia and hyperinsulinemia caused by IR may reverse this gender protection. Studies have shown that all insulin replacement markers have a good correlation with HOMA-IR of both sexes, and the correlation between female is stronger than that of male [40]. However, some studies have found that there was no gender difference between the TyG index and MACEs in patients with hypertension [41]. There is an investigation of the association between the TyG index and the early stages of subclinical atherosclerosis (SA) between the sexes. A high TyG index is independently associated with SA in non-diabetic female, but in NGT male. Regardless of

Page $18 / 25$ 
gender, TyG index has nothing to do with the presence of SA in diabetic patients [42]. Not only in DM patients, but also in NGT participants, the higher TG and blood pressure have a greater impact, and the number of CVDs events and deaths in female was higher than that in male [43]. Consistent with the results of this study, female's Tyg index had a higher correlation with CAP than male, and a high level of Tyg index had a higher correlation with CAP.

A study showed that in the middle-aged and elderly population, the increase in TyG index was significantly associated with hypertension and isolated systolic hypertension [44]. This study shows that the OR value of middle-aged patients with CHD is higher than that of elderly patients with CHD. TyG index was significantly related to the risk of CAP in middle-aged patients with NGT, while TyG index is significantly related to the risk of CAP in elderly patients with DM. It may be because this study is a CHD population, the average age is over 60 years old, belongs to the middle-aged and elderly population, and there is a certain bias for age. Therefore, in the future research, people of different ages can be included and studied to the correlation between Tyg index and CAP under different ages.

To sum up, with more and more studies on the influence of TyG index on patients with cardiovascular diseases, the clinical significance of TyG index is becoming more and more clear. The evaluation of TyG index may have important clinical significance for risk stratification and individualized treatment of these CHD patients.

\section{Strengths and limitations}

This study is a large-scale multicenter cohort study, but it has several limitations. First of all, this study is a multi-center study, and there may be bias in the measurement methods of different research centers. However, because the external quality assessment between clinical laboratories in each center is conducted by practitioners. Secondly, this study is a cross-sectional study. Therefore, we can't establish causality according to the results of this study. The exact mechanism of the relationship between TyG index and CAP needs further prospective large-scale research.

\section{Conclusion}

This study had proved the significant correlation between TyG index and CAP of CHD patients. In addition, the correlation between TyG index and CAP in CHD patients was higher in female than in male, and higher in middle-aged and elderly than in the elderly. Under the condition of DM, the correlation between TyG index and CAP in CHD patients was higher. As a marker of IR, TyG index is easy to calculate, and it may reflect the risk of CAP in CHD patients. And the results of this study may emphasize the need for a risk management strategy for specific gender and different age groups to prevent the occurrence of CAP in CHD patients.

\section{Abbreviations}

TyG: triglyceride glucose

CHD: coronary heart disease

DM: diabetes 
NCD: non-communicable diseases

T2DM: Type 2 diabetes

CAD: coronary artery disease

CAP: carotid artery plaque

CIMT: carotid artery intima-media thickness

Pre-DM: prediabetes

AS: atherosclerosis

SBP: systolic blood pressure

DBP: diastolic blood pressure

FPG: fasting plasma glucose

TC: total cholesterol

HDL-C: high-density lipoprotein cholesterol

TG: triglycerides

LDL-C: low-density lipoprotein cholesterol

CRP: C-reactionprotein

HbA1c: glycated haemoglobin

OR: odds ratios

HOMA-IR: homeostasis model assessment of insulin resistance

SA: subclinical atherosclerosis

\section{Declarations}

\section{Authors' contributions}

CQY, GS, HMZ, and ZL participated in the study design and statistical analysis; ZL and YYH analysed the data together and drafted the article; SW, LL, RRY, YJL, QC, LY, and YCZ participated in data collection. All authors read and approved the final manuscript.

\section{Acknowledgments}

We thank all the participants in the study and the members of the survey teams, as well as the financial support. 


\section{Declaration of conflict of interest}

The authors declare no competing interests.

\section{Funding}

This work was supported by the National Basic Research Program of China (973 project, grant numbers: 2014CB542902).

\section{Availability of data and materials}

The datasets used and/or analyzed in the current study are available from the corresponding author upon reasonable request.

\section{Ethics approval and consent to participate}

This study was approved by the ethics committee of Tianjin University of Traditional Chinese Medicine (TJUTCM-EC20190008) and registered with the Chinese Clinical Trial Registry (ChiCTR-1900024535) and in Clinical Trials.gov ( NCT04026724).

\section{Consent for publication}

Not applicable.

\section{References}

1. Wang AL, Zhang H, Zhang J, Zhang Y, Cao HJ, Liu JP, Xu H, Chen KJ. Adjuvant Effects of Health Education of Chinese Medicine for Chronic Diseases: A Systematic Review and Meta-Analysis of Randomized Controlled Trials. Evid Based Complement Alternat Med. 2020;2020:3738753. doi: 10.1155/2020/3738753.

2. Zhang, MY, Zhang, JH, Chua, HZ, Feng R, Lu, MJ, Tian Y. Core outcome set for stable angina pectoris in traditional Chinese medicine (COS-SAP-TCM). Acupuncture and Herbal Medicine. 2021;1(1):39-48. doi: 10.1097/HM9.0000000000000007

3. Zhao, Q, Cheng, YJ, Xu, YK, Zhao, ZW, Liu, C, Sun, TN, \& Zhou, YJ. Comparison of various insulin resistance surrogates on prognostic prediction and stratification following percutaneous coronary intervention in patients with and without type 2 diabetes mellitus. Cardiovascular diabetology. 2021;20(1):190. https://doi.org/10.1186/s12933-021-01383-7

4. Juárez-Rojas JG, Posadas-Romero C, Martínez-Alvarado R, Jorge-Galarza E, Reyes-Barrera J, SánchezLozada LG, Torres-Tamayo M, Medina-Urrutia AX. Type 2 Diabetes Mellitus is Associated with Carotid Artery Plaques in Patients with Premature Coronary Heart Disease. Rev Invest Clin. 2018;70(6):301-309. doi: 10.24875/RIC.18002591.

5. Wang L, Gao P, Zhang M, Huang Z, Zhang D, Deng Q, Li Y, Zhao Z, Qin X, Jin D, Zhou M, Tang X, Hu Y, Wang L. Prevalence and Ethnic Pattern of Diabetes and Prediabetes in China in 2013. JAMA. 2017;317(24):25152523. doi: 10.1001/jama.2017.7596.

6. Jin JL, Cao YX, Liu HH, Zhang HW, Guo YL, Wu NQ, Zhu CG, Xu RX, Gao Y, Sun J, Dong Q, Li JJ. Impact of free fatty acids on prognosis in coronary artery disease patients under different glucose metabolism 
status. Cardiovasc Diabetol. 2019;18(1):134. doi: 10.1186/s12933-019-0936-8.

7. Liu HH, Cao YX, Li S, Guo YL, Zhu CG, Wu NQ, Gao Y, Dong QT, Zhao X, Zhang Y, Sun D, Li JJ. Impacts of Prediabetes Mellitus Alone or Plus Hypertension on the Coronary Severity and Cardiovascular Outcomes. Hypertension.2018;71(6):1039-1046.doi:10.1161/HYPERTENSIONAHA.118.11063.

8. Jin JL, Cao YX, Zhang HW, Sun D, Hua Q, Li YF, Guo YL, Wu NQ, Zhu CG, Gao Y, Dong QT, Liu HH, Dong Q, Li JJ. Lipoprotein(a) and Cardiovascular Outcomes in Patients With Coronary Artery Disease and Prediabetes or Diabetes. Diabetes Care. 2019;42(7):1312-1318. doi: 10.2337/dc19-0274.

9. Chamroonkiadtikun P, Ananchaisarp T, Wanichanon W. The triglyceride-glucose index, a predictor of type 2 diabetes development: A retrospective cohort study. Prim Care Diabetes. 2020;14(2):161-167. doi:10.1016/j.pcd.2019.08.004.

10. Alizargar J, Bai CH, Hsieh NC, Wu SV. Use of the triglyceride-glucose index (TyG) in cardiovascular disease patients. Cardiovasc Diabetol. 2020;19(1):8. doi: 10.1186/s12933-019-0982-2.

11. da Silva A, Caldas APS, Hermsdorff HHM, Bersch-Ferreira ÂC, Torreglosa CR, Weber B, Bressan J. Triglyceride-glucose index is associated with symptomatic coronary artery disease in patients in secondary care. Cardiovasc Diabetol. 2019;18(1):89. doi: 10.1186/s12933-019-0893-2.

12. Luo E, Wang D, Yan G, Qiao Y, Liu B, Hou J, Tang C. High triglyceride-glucose index is associated with poor prognosis in patients with acute ST-elevation myocardial infarction after percutaneous coronary intervention. Cardiovasc Diabetol. 2019;18(1):150. doi: 10.1186/s12933-019-0957-3.

13. Shi W, Xing L, Jing L, Tian Y, Yan H, Sun Q, Dai D, Shi L, Liu S. Value of triglyceride-glucose index for the estimation of ischemic stroke risk: Insights from a general population. Nutr Metab Cardiovasc Dis. 2020;30(2):245-253. doi: 10.1016/j.numecd.2019.09.015.

14. Zhao S, Yu S, Chi C, Fan X, Tang J, Ji H, Teliewubai J, Zhang Y, Xu Y. Association between macro- and microvascular damage and the triglyceride glucose index in community-dwelling elderly individuals: the Northern Shanghai Study. Cardiovasc Diabetol. 2019;18(1):95. doi: 10.1186/s12933-019-0898-x.

15. Lee SB, Ahn CW, Lee BK, Kang S, Nam JS, You JH, Kim MJ, Kim MK, Park JS. Association between triglyceride glucose index and arterial stiffness in Korean adults. Cardiovasc Diabetol. 2018;17(1):41. doi: 10.1186/s12933-018-0692-1.

16. Kim MK, Ahn CW, Kang S, Nam JS, Kim KR, Park JS. Relationship between the triglyceride glucose index and coronary artery calcification in Korean adults. Cardiovasc Diabetol. 2017;16(1):108. doi: 10.1186/s12933-017-0589-4.

17. Jian S, Su-Mei N, Xue C, Jie Z, Xue-Sen W. Association and interaction between triglyceride-glucose index and obesity on risk of hypertension in middle-aged and elderly adults. Clin Exp Hypertens. 2017;39(8):732739. doi: 10.1080/10641963.2017.1324477.

18. Lee EY, Yang HK, Lee J, Kang B, Yang Y, Lee SH, Ko SH, Ahn YB, Cha BY, Yoon KH, Cho JH. Triglyceride glucose index, a marker of insulin resistance, is associated with coronary artery stenosis in asymptomatic subjects with type 2 diabetes. Lipids Health Dis. 2016;15(1):155. doi: 10.1186/s12944-016-0324-2.

19. Irace C, Carallo C, Scavelli FB, De Franceschi MS, Esposito T, Tripolino C, Gnasso A. Markers of insulin resistance and carotid atherosclerosis. A comparison of the homeostasis model assessment and triglyceride glucose index. Int J Clin Pract. 2013;67(7):665-72. doi: 10.1111/ijcp.12124. 
20. Barua RS, Rigotti NA, Benowitz NL, Cummings KM, Jazayeri MA, Morris PB, Ratchford EV, Sarna L, Stecker EC, Wiggins BS. 2018 ACC Expert Consensus Decision Pathway on Tobacco Cessation Treatment: A Report of the American College of Cardiology Task Force on Clinical Expert Consensus Documents. J Am Coll Cardiol. 2018;72(25):3332-3365. doi: 10.1016/j.jacc.2018.10.027.

21. Fan AZ, Ruan WJ, Chou SP. Re-examining the relationship between alcohol consumption and coronary heart disease with a new lens. Prev Med. 2019;118:336-343. doi: 10.1016/j.ypmed.2018.11.022.

22. Flack JM, Adekola B. Blood pressure and the new ACC/AHA hypertension guidelines. Trends CardiovasC Med. 2020;30(3):160-164. doi: 10.1016/j.tcm.2019.05.003.

23. Wu S, Xu L, Wu M, Chen S, Wang Y, Tian Y. Association between triglyceride-glucose index and risk of arterial stiffness: a cohort study. Cardiovasc Diabetol. 2021;20(1):146. doi: 10.1186/s12933-021-01342-2.

24. Zhu JR, Gao RL, Zhao SP, Lu GP, Zhao D, Li JJ. Guidelines for the prevention and treatment of dyslipidemia in Chinese adults (Revised Edition 2016), J Chi Circ. 2016;31(10):937-953.

25. Yang L, Li Z, Song Y, Liu Y, Zhao H, Liu Y, Zhang T, Yuan Y, Cai X, Wang S, Wang P, Gao S, Li L, Li Y, Yu C. Study on urine metabolic profiling and pathogenesis of hyperlipidemia. Clin Chim Acta. 2019;495:365-373. doi: 10.1016/j.cca.2019.05.001.

26. Liu Y, Zhu Y, Jia W, Sun D, Zhao L, Zhang C, Wang C, Chen G, Fu S, Bo Y, Xing Y. Association between lipid profiles and presence of carotid plaque. Sci Rep. 2019;9(1):18011. doi: 10.1038/s41598-019-54285-w.

27. Lee SH, Kwon HS, Park YM, Ha HS, Jeong SH, Yang HK, Lee JH, Yim HW, Kang MI, Lee WC, Son HY, Yoon $\mathrm{KH}$. Predicting the development of diabetes using the product of triglycerides and glucose: the Chungju Metabolic Disease Cohort (CMC) study. PLoS One. 2014;9(2):e90430. doi: 10.1371/journal.pone.0090430.

28. Irace C, Carallo C, Scavelli FB, De Franceschi MS, Esposito T, Tripolino C, Gnasso A. Markers of insulin resistance and carotid atherosclerosis. A comparison of the homeostasis model assessment and triglyceride glucose index. Int J Clin Pract. 2013;67(7):665-72. doi: 10.1111/ijcp.12124.

29. Won KB, Lee BK, Park HB, Heo R, Lee SE, Rizvi A, Lin FY, Kumar A, Hadamitzky M, Kim YJ, Sung JM, Conte E, Andreini D, Pontone G, Budoff MJ, Gottlieb I, Chun EJ, Cademartiri F, Maffei E, Marques H, de Araújo Gonçalves P, Leipsic JA, Shin S, Choi JH, Virmani R, Samady H, Chinnaiyan K, Raff GL, Stone PH, Berman DS, Narula J, Shaw LJ, Bax JJ, Min JK, Chang HJ. Quantitative assessment of coronary plaque volume change related to triglyceride glucose index: The Progression of AtheRosclerotic PIAque Determlned by Computed TomoGraphic Angiography IMaging (PARADIGM) registry. Cardiovasc Diabetol. 2020;19(1):113. doi: 10.1186/s12933-020-01081-w.

30. Zhang Y, Ding X, Hua B, Liu Q, Gao H, Chen H, Zhao XQ, Li W, Li H. High triglyceride-glucose index is associated with adverse cardiovascular outcomes in patients with acute myocardial infarction. Nutr Metab Cardiovasc Dis. 2020;30(12):2351-2362. doi: 10.1016/j.numecd.2020.07.041.

31. Wu Z, Zhou D, Liu Y, Li Z, Wang J, Han Z, Miao X, Liu X, Li X, Wang W, Guo X, Tao L. Association of TyG index and TG/HDL-C ratio with arterial stiffness progression in a non-normotensive population. Cardiovasc Diabetol. 2021;20(1):134. doi: 10.1186/s12933-021-01330-6.

32. Park K, Ahn CW, Lee SB, Kang S, Nam JS, Lee BK, Kim JH, Park JS. Elevated TyG Index Predicts Progression of Coronary Artery Calcification. Diabetes Care. 2019;42(8):1569-1573. doi: 10.2337/dc181920. 
33. Ormazabal V, Nair S, Elfeky O, Aguayo C, Salomon C, Zuñiga FA. Association between insulin resistance and the development of cardiovascular disease. Cardiovasc Diabetol. 2018;17(1):122. doi:

10.1186/s12933-018-0762-4.

34. Nam KW, Kwon HM, Jeong HY, Park JH, Kwon H, Jeong SM. High triglyceride-glucose index is associated with subclinical cerebral small vessel disease in a healthy population: a cross-sectional study. Cardiovasc Diabetol. 2020;19(1):53. doi: 10.1186/s12933-020-01031-6.

35. Liu J, Rutten-Jacobs L, Liu M, Markus HS, Traylor M. Causal Impact of Type 2 Diabetes Mellitus on Cerebral Small Vessel Disease: A Mendelian Randomization Analysis. Stroke. 2018;49(6):1325-1331. doi: 10.1161/STROKEAHA.117.020536.

36. Shi W, Xing L, Jing L, Tian Y, Yan H, Sun Q, Dai D, Shi L, Liu S. Value of triglyceride-glucose index for the estimation of ischemic stroke risk: Insights from a general population. Nutr Metab Cardiovasc Dis. 2020;30(2):245-253. doi: 10.1016/j.numecd.2019.09.015.

37. da Silva A, Caldas APS, Rocha DMUP, Bressan J. Triglyceride-glucose index predicts independently type 2 diabetes mellitus risk: A systematic review and meta-analysis of cohort studies. Prim Care Diabetes. 2020;14(6):584-593. doi: 10.1016/j.pcd.2020.09.001.

38. Emerging Risk Factors Collaboration, Sarwar N, Gao P, Seshasai SR, Gobin R, Kaptoge S, Di Angelantonio E, Ingelsson E, Lawlor DA, Selvin E, Stampfer M, Stehouwer CD, Lewington S, Pennells L, Thompson A, Sattar N, White IR, Ray KK, Danesh J. Diabetes mellitus, fasting blood glucose concentration, and risk of vascular disease: a collaborative meta-analysis of 102 prospective studies. Lancet. 2010;375(9733):2215-22. doi: 10.1016/S0140-6736(10)60484-9.

39. Ariel D, Reaven G. Modulation of coronary heart disease risk by insulin resistance in subjects with normal glucose tolerance or prediabetes. Acta Diabetol. 2014;51(6):1033-9. doi: 10.1007/s00592-014-0667-y.

40. Nakagomi A, Sunami Y, Kawasaki Y, Fujisawa T, Kobayashi Y. Sex difference in the association between surrogate markers of insulin resistance and arterial stiffness. J Diabetes Complications. 2020;34(6):107442. doi: 10.1016/j.jdiacomp.2019.107442.

41. Yang K, Liu W. Triglyceride and Glucose Index and Sex Differences in Relation to Major Adverse Cardiovascular Events in Hypertensive Patients Without Diabetes. Front Endocrinol (Lausanne). 2021;12:761397. doi: 10.3389/fendo.2021.761397.

42. Lu YW, Chang CC, Chou RH, Tsai YL, Liu LK, Chen LK, Huang PH, Lin SJ. Gender difference in the association between TyG index and subclinical atherosclerosis: results from the I-Lan Longitudinal Aging Study. Cardiovasc Diabetol. 2021;20(1):206. doi: 10.1186/s12933-021-01391-7.

43. Tramunt B, Smati S, Grandgeorge N, Lenfant F, Arnal JF, Montagner A, Gourdy P. Sex differences in metabolic regulation and diabetes susceptibility. Diabetologia. 2020;63(3):453-461. doi: 10.1007/s00125019-05040-3.

44. Zheng R, Mao Y. Triglyceride and glucose (TyG) index as a predictor of incident hypertension: a 9-year longitudinal population-based study. Lipids Health Dis. 2017;16(1):175. doi: 10.1186/s12944-017-0562-y.

\section{Figures}




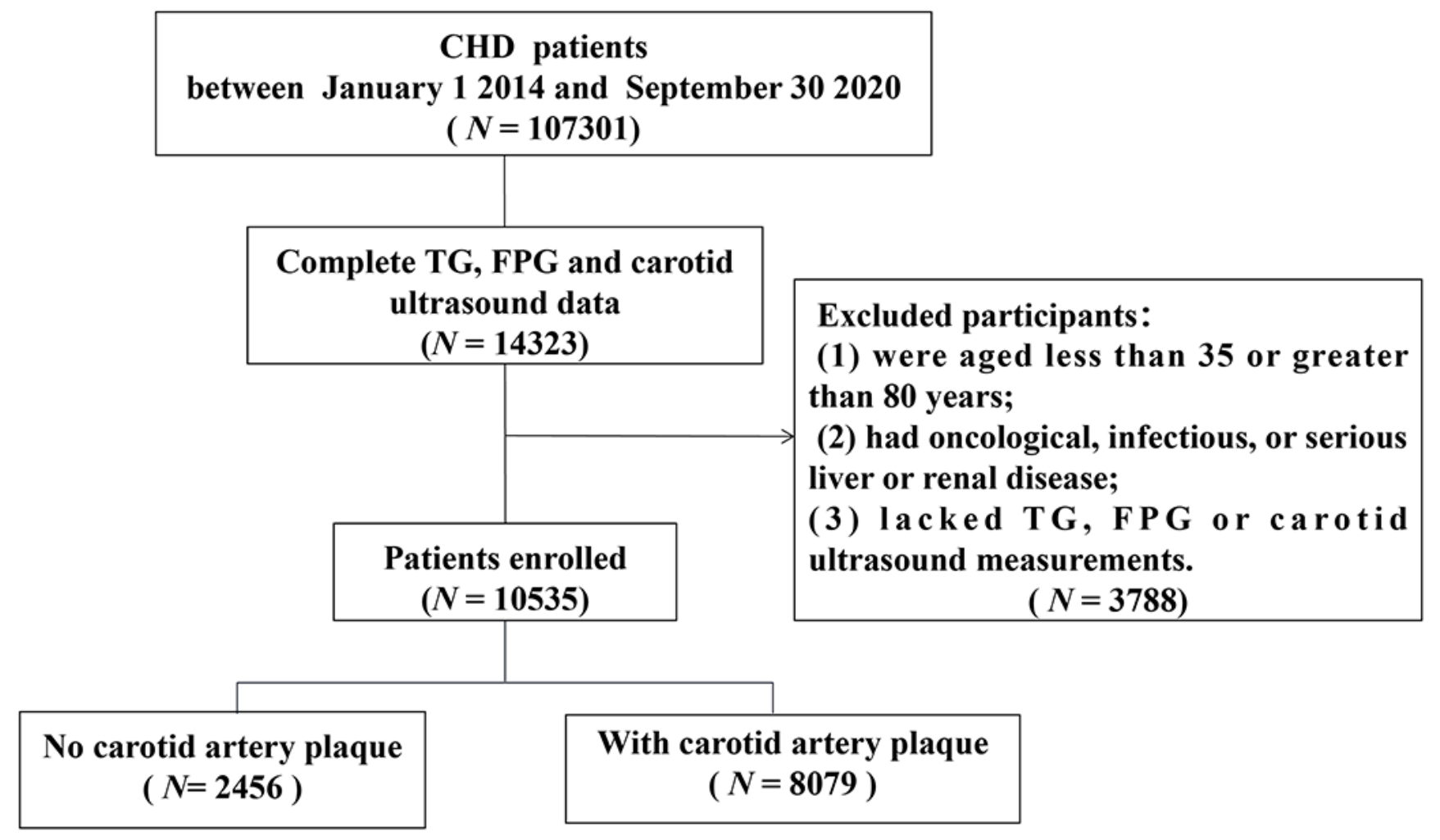

Figure 1

Flow chart of patient recruitment

\section{Supplementary Files}

This is a list of supplementary files associated with this preprint. Click to download.

- Supplementarymaterial.docx

- Graphicalabstract.tif 